\title{
Características prosociales y producción científica de académicos de la Facultad de Filosofía y Letras de la Universidad Autónoma de Chihuahua
}

\author{
The prosocial characteristics and scientific production of professors from the \\ Facultad de Filosofía y Letras from the Universidad Autónoma de Chihuahua
}

Doris Agredo-Machin • Sarah Margarita Chávez-Valdez • José Refugio Romo-González

Doris Agredo-Machin. Universidad Autónoma de Chihuahua, México. Es maestrante en Innovación Educativa (beca CONACYT) en la UACH y licenciada en Psicología por la Escuela Libre de Psicología-UCC. Sus líneas de investigación incluyen psicología social, actitudes prosociales y su influencia sobre la producción científica. Correo electrónico:dagredo@gmail.com. ORCID: https://orcid.org/0000-0003-0132-0404.

Sarah Margarita Chávez-Valdez. Escuela Libre de Psicología, A.C., Chihuahua, México. Es investigadora principal y catedrática en licenciatura y maestría desde 2007 en la Escuela Libre de Psicología-Universidad de Ciencias del Comportamiento, miembro del Sistema Nacional de Investigadores. Sus líneas de investigación incluyen psicología social y de la salud, violencia juvenil, abuso de sustancias, victimización, agresión, maltrato y exposición social a la violencia. Ha realizado revisiones para varias revistas científicas de su área. Correo electrónico: sarahmargaritachavezv@gmail.com. ORCID: https://orcid. org/0000-0003-0714-5379.

José Refugio Romo-González. Universidad Autónoma de Chihuahua, México.
Resumen

Este artículo resume los hallazgos de una investigación cuantitativa y correlacional que tuvo el propósito de relacionar diversos rasgos de conductas prosociales con la producción científica de una muestra de 31 profesores con el grado de doctor de la Facultad de Filosofía y Letras de la Universidad Autónoma de Chihuahua. Se empleó una encuesta con 73 reactivos evaluados con una escala de tipo Likert de cinco puntos, los cuales se clasificaron bajo seis dimensiones de características prosociales: a) motivación intrínseca, b) motivación extrínseca, c) autoestima, d) empatía, e) autorregulación y f) conducta prosocial. El análisis de datos se realizó en función de datos sociodemográficos, de perfil profesional, los distintos productos científicos que acreditaron los docentes y las seis dimensiones prosociales. Entre los resultados más relevantes se encontró que: I) los doctores con mayor producción global obtuvieron calificaciones más bajas en las seis dimensiones prosociales; II) los investigadores más jóvenes son quienes han publicado la mayor cantidad de artículos y obtuvieron las mejores puntuaciones en las características prosociales; III) los investigadores que tienen la mayor cantidad global de producción científica tuvieron una calificación en motivación intrínseca ligeramente superior; IV) quienes obtuvieron su grado de doctor en el extranjero concentran más de la mitad de la producción (seis de ellos están en el SNI), y v) los miembros del SNI también concentran más de la mitad de la producción.

Palabras clave: Aspectos psicológicos, personalidad, profesores, publicaciones científicas.

\section{Abstract}

This article summarizes the results of a quantitative and correlational research that sought to relate diverse prosocial behavior traits with the scientific production 
Es Miembro del Sistema Nacional de Investigadores y Perfil Prodep. Doctorado en Administración por la UACH y maestría en Gestión de la Información y el Conocimiento por la Universitat Oberta de Catalunya (España). Profesor-investigador desde 1991 en la Facultad de Filosofía y Letras (UACH), integrante del núcleo académico básico del doctorado en Educación, Artes y Humanidades y en la maestría en Innovación Educativa (ambos PNPC). Miembro del Cuerpo Académico Consolidado "Estudios de la Información". Correo electrónico: jromo@uach.mx. ORCID: https:// orcid.org/0000-0002-4810-4357. of a sample of 31 professors with a doctoral degree from the Facultad de Filosofía y Letras at the Universidad Autónoma de Chihuahua. A survey of 73 items that uses a five-point Likert-type scale to rate each one was employed, classifying them in six dimensions of prosocial characteristics: a) intrinsic motivation, b) extrinsic motivation, c) self-esteem, d) empathy, e) self-regulation, and f) prosocial behavior. The data was analyzed by considering sociodemographic characteristics, professional profiles, the diverse scientific products accredited by the professors and the six prosocial dimensions. Among the most relevant results, it was found that: I) the doctors with the largest global production qualified lower in the six prosocial dimensions; II) the younger researchers have published the largest number of articles and obtained the best scores in the prosocial characteristics; III) the researchers with the largest global production qualified slightly higher in intrinsic motivation; IV) those who obtained their doctoral degree outside of Mexico concentrate more than half of the production and six of them are SNI members, and v) SNI members also concentrate more than half of the production.

Keywords: Psychological aspects, personality, professors, scientific publications.

\section{INTRODUCCIÓN}

Esta investigación cuantitativa de corte correlacional buscó relacionar diversos rasgos de conductas prosociales con las distintas manifestaciones de la producción científica. Por lo tanto, esta investigación se relacionó con la importancia de indagar las razones por las que se presenta un déficit de producción científica (Martínez, 2018), a pesar de la existencia de distintos estímulos gubernamentales que intentan disminuir la incesante demanda de investigadores que contribuyan al conocimiento de diversas áreas (Sánchez, 2017). Esto llevó a explorar variables asociadas a factores psicológicos que puedan inhibir, fomentar o facilitar la productividad científica de los docentes (Landini, 2015; Landini, Olivera y De Hegedüs, 2017). Asimismo, se revisó el número de artículos publicados y el surgimiento de las políticas públicas creadas para generar estímulos económicos que fomentasen la investigación (Rodríguez, 2016; Inzunza, 2017).

La revisión de la literatura especializada ayudó a generar los ítems que se integraron en una encuesta, por lo cual estos fueron sustentados por distintas teorías e investigaciones pertinentes. Los temas principales de dicha revisión fueron: motivación, locus de control, autoestima, empatía, autorregulación, actitud prosocial, evaluación de productividad científica, motivación detrás de la investigación científica, investigadores altamente productivos, preocupaciones de los investigadores y el valor de la colaboración.

\section{Metodología}

Se realizó un análisis estadístico de los datos recabados mediante una encuesta como instrumento de recolección de datos, permitiendo así un análisis deductivo. El diseño 
de investigación fue de tipo correlacional, exploratoria y no experimental. Se buscó indagar sobre las variables independientes, entendidas como los rasgos personales de los docentes: a) autoestima; b) autorregulación, dentro de la cual encontramos la motivación y locus de control, y c) conductas prosociales. Las variables dependientes comprendieron los efectos, en este caso los productos científicos de los docentes. Esto se hizo con la finalidad de encontrar correlaciones entre los aspectos psicológicos y la producción científica, para analizar las relaciones entre ambos. Una vez clasificadas las variables, se plantearon las hipótesis a confirmar o rechazar: I) los investigadores que tienen mejores características prosociales tendrán una mayor producción científica; II) los investigadores que tienen buena producción científica tendrán una mayor motivación intrínseca, asociada al locus de control interno; III) los investigadores jóvenes son más productivos que aquellos más experimentados; IV) los investigadores que tienen formación en el extranjero poseen una mayor cantidad de producción científica, y v) los miembros del Sistema Nacional de Investigadores (SNI) son quienes concentran la mayor parte de la producción.

Los objetivos incluyeron explorar las características de las variables independientes y las variables dependientes, para comprender sus interrelaciones, así como determinar las variables asociadas a las conductas prosociales y los factores psicológicos que pudiesen inhibir, fomentar o facilitar la productividad científica del investigador, a través de la elaboración de un inventario que permitiese medir la intensidad, presencia o ausencia de estos factores, para así indagar en las posibles correlaciones existentes entre variables y determinar la existencia o no de diferencias significativas. La muestra fue seleccionada entre los académicos con grado de doctor adscritos a la Facultad de Filosofía y Letras (FFyL) de la Universidad Autónoma de Chihuahua (UACH), lo cual resultó en que 31 de los 47 profesores participaron en la encuesta, gracias a la información proporcionada por la Secretaría de Investigación y Posgrado de la FFyL de la UACH.

Instrumento de recolección de datos

Se diseñó y empleó una encuesta dividida en seis partes. En la primera parte se realizaron preguntas generales (sobre segmentación sociodemográfica y perfil profesional) de los participantes, tales como: sexo, edad, área del doctorado y su país de obtención, el número de años de experiencia en docencia y en investigación, si poseen el perfil del Programa para el Desarrollo Profesional Docente (Proder), si pertenecen o no a un grupo disciplinar, cuerpo académico o al SNI. De la segunda a la quinta parte del instrumento se incluyeron 73 ítems para ser calificados empleando una escala de tipo Likert de cinco puntos, con el fin de usar una única escala para que las respuestas fueran comparables entre sí, obtener los estadísticos entre secciones, aplicar correlaciones y poder calcular el alfa de Cronbach. Estos 73 reactivos, basados en las teorías y resultados de investigación disponibles en las fuentes especializadas que se estudiaron, se clasificaron temáticamente en varias secciones de la encuesta, que 
son presentadas a continuación: actitudes prosociales de los encuestados, razones por las cuales investigar; factores que estimulan positivamente la propia investigación y producción científica; apreciación sobre el propio desempeño o disposición y en comparación con sus pares. Además, los 73 reactivos se emplearon para calcular seis nuevas variables, correspondientes a las seis dimensiones utilizadas para analizar los datos: a) motivación intrínseca, b) motivación extrínseca, c) autoestima, d) empatía, e) autorregulación y f) conducta prosocial. La conformación de estas dimensiones resultó de la suma de los reactivos pertenecientes a cada dimensión que se presentaron a lo largo del instrumento, lo cual permitió apreciar el efecto lineal y aditivo.

La segunda parte de la encuesta midió las actitudes prosociales de los encuestados, empleando los reactivos de Caprara, Steca, Zelli y Capanna (2005), los cuales fueron insertados dentro del instrumento empleado, debido a la complejidad de la medición de las características prosociales según los autores citados, por lo cual se escogió incorporar estos reactivos a nuestro instrumento, el cual fue validado en varias aplicaciones con adultos. Estos reactivos son aplicables a la investigación de las características prosociales en adultos y sus 16 ítems están asociados con cuatro acciones: "compartir, ayudar, cuidar, y sentirse empático con los demás y sus necesidades o solicitudes" (Caprara et al., 2005, p. 80).

La tercera sección consistió en un inventario sobre las razones por las cuales se realizan actividades de investigación, las cuales se fundamentaron en fuentes especializadas:

I. Para el avance de mi disciplina (Ladd, Lappé, McCormick, Boyce y Cho, 2009; Swan y Brown, 2005; Öcshner, 2013).

II. Para el avance de la sociedad y la humanidad (Ladd et al., 2009; Swan y Brown, 2005; Öcshner, 2013).

III. Para mejorar la reputación de mi institución (Swan y Brown, 2005).

IV. Para formar nuevos investigadores (Offutt, 2011).

V. Para compartir nuevo conocimiento con mis pares (Öcshner, 2013).

VI. Para avanzar en mi carrera, ser evaluado positivamente y enriquecer mi currículum (Wigfield, 2002; Swan y Brown, 2005; Mulligan y Mabe, 2011; Offutt, 2011; Mattedi y Spiess, 2017).

VII. Reclamar para mí mismo mi propio trabajo y documentar mis resultados de investigación para la posteridad (Swan y Brown, 2005).

VIII. Por requisitos laborales o por obligación (Swan y Brown, 2005).

IX. Para obtener retroalimentación de mis pares y de la comunidad académica (Swan y Brown, 2005).

X. Para encontrar mayores oportunidades de vinculación y colaboración con mis pares (Landini, Olivera y De Hegedüs, 2017; Siciliano, Welch y Feeney, 2018).

XI. Para obtener fondos y apoyos para continuar investigando (Offutt, 2011; Mulligan y Mabe, 2011; Öcshner, 2013). 
XII. Para ejercer influencia y liderazgo en mi campo de investigación (Offutt, 2011; Landini, Olivera y De Hegedüs, 2017).

XIII. Para obtener mayor prestigio y reputación (Mattedi y Spiess, 2017).

XIV. Para obtener compensaciones económicas adicionales a mi sueldo (Öcshner, 2013).

XV. Por satisfacción personal, interés y disfrute (Wigfield, 2002; Swan y Brown, 2005).

En la cuarta sección, los encuestados debían calificar una serie de factores que afectan sus actividades de investigación y producción científica. Los factores a calificar fueron los siguientes: I) estímulos para realizar investigación; II) reconocimiento de estas labores por parte de mi institución; III) autonomía y libertad para organizar y hacer mi trabajo en función de las actividades asignadas; IV) provisión de recursos (presupuestales y de equipamiento) para realizar investigación; v) disposición de entornos creativos; VI) disponibilidad de colegas con quienes colaborar; VII) disposición de tiempo; VIII) condiciones de seguridad y salud; IX) carga laboral; x) organización administrativa de mi institución; XI) oferta en capacitación sobre investigación; XII) nivel de objetividad de los sistemas de evaluación de investigadores (Öcshner, 2013; Reyes y Perales, 2016).

La quinta sección se dividió en dos partes, la primera evaluó la autorregulación de los participantes, según aspectos recuperados en las fuentes consultadas, incluyendo preguntas relacionadas con la autoeficacia, autorregulación y con la motivación intrínseca. Incluimos esta sección en el instrumento ya que los investigadores exitosos deben tener un sentido del propósito, autoeficacia, autocontrol de sus avances y un buen nivel de satisfacción en el trabajo (Schultz, Jones-Walker y Chikkatur, 2008). En cuanto a la medición de la autoeficacia, frecuentemente se realiza preguntando al sujeto directamente si cree que puede realizar la tarea en cuestión (Pajares, 1996, citado por Wigfield, 2002). Bandura (1997) también sostiene que las preguntas deben realizarse sobre las creencias específicas del individuo, ya que estas se relacionan con su comportamiento. Por lo tanto, para medir autoeficacia hemos incluido preguntas que han sido derivadas de varios autores, pero orientadas al área de la investigación y producción científica. Dichas preguntas invitaron al sujeto a responder sobre sus creencias en cuanto a sus capacidades para realizar actividades relacionadas con la investigación y producción científica. Las capacidades de autoeficacia sobre las que indagó el instrumento incluyeron: I) puedo hacer lo que sea necesario para investigar; II) puedo realizar una investigación científica; III) puedo publicar mi investigación en una revista científica arbitrada e indizada; IV) soy capaz y puedo aprender lo que no sé en cuanto a la investigación; v) me falta mucho por aprender; vI) puedo decidir sobre un tema para investigar; VII) puedo formular una pregunta de investigación; VIII) puedo obtener una evaluación positiva como investigador; IX) puedo superar las barreras externas que afectan mis labores de investigación (Bandura, 1997; Wigfield, 2002; Cardelle-Elawar y Sanz, 2010). Las afirmaciones relacionadas con la autorre- 
gulación que se incluyeron en esta misma sección fueron: $x$ ) puedo establecer planes y metas de investigación, los cuales cumplo en tiempo y forma; xI) puedo hacer seguimiento y evaluar mis avances de investigación alcanzados (Patterson-Hazley y Kiewra, 2013). Las afirmaciones que, si bien tuvieron que ver con características del individuo en cuanto a su autoeficacia, también se relacionaban con la motivación intrínseca, incluyeron: XII) soy capaz de sacrificar mi tiempo libre para investigar; XIII) puedo priorizar mi actividad investigativa por encima de mis relaciones familiares y/o personales; XIV) puedo realizar un autodiagnóstico de mis capacidades para mejorarlas, y xv) puedo perseverar a pesar de las dificultades y enfocarme al logro de mis actividades de investigación (Reyes y Perales, 2016).

La segunda parte de la quinta sección solicitó a los encuestados que se compararan con sus pares en cuanto a su autoeficacia, autorregulación y motivación intrínseca, por lo cual se utilizaron los mismos reactivos que en la primera parte, pero con ajustes en su redacción. Finalmente, la última sección de la encuesta buscó obtener datos sobre los productos de investigación de los encuestados de los últimos tres años (2016-2018).

\section{Resultados}

Los ítems del instrumento utilizado se basaron en fuentes especializadas que brindan validez teórica. Además, con las respuestas obtenidas obtuvimos buenos niveles en el cálculo del alfa de Cronbach en las distintas secciones del instrumento, lo cual brinda consistencia interna y confiabilidad. El alfa de la segunda sección de la encuesta (conductas prosociales), que corresponde a los reactivos de Caprara et al. (2005), resultó de 0.934; el de la tercera sección (razones por las cuales investigar) fue de 0.874; el de la cuarta (factores que estimulan positivamente la propia investigación) resultó en 0.892; la quinta sección (autorregulación) fue subdividida en dos partes, el alfa de la primera (apreciación hacia el propio desempeño o disposición) fue de 0.893 y el de la segunda (comparación con sus pares) fue de 0.934. Finalmente, el alfa de los 73 ítems del instrumento, repartidos a lo largo de las cinco secciones, fue de 0.945 , valor que se considera excelente para determinar la consistencia interna y confiabilidad entre las puntuaciones y los ítems en cuestión.

Entre las variables producción científica y las variables sexo, área o país, no se encontraron correlaciones; en cambio, se encontraron dos correlaciones significativas entre las variables de producción científica y edad, pero de nivel de asociación muy bajo, estas fueron con la publicación de libros $(\mathrm{r}=0.355$, sig $=0.050)$ y de capítulos de libros $(r=0.361$, sig $=0.046)$. Esto indica que, a mayor edad, los productos que los doctores podrían tener en mayor abundancia son libros y capítulos de libros, aunque la posibilidad de que esto ocurra es muy baja. Lo interesante de que la edad no tenga tanto que ver con la producción es que estos profesores producen sin que su edad sea crucial.

Entre productos científicos, se encontró que existen correlaciones muy significativas $(\operatorname{sig}=0.000)$ y de nivel de asociación de alto a muy alto entre la 'suma de productos 
científicos' y los artículos $(\mathrm{r}=0.711)$, así como con los artículos en colaboración $(\mathrm{r}=$ $0.643)$, los proyectos $(\mathrm{r}=0.552)$, libros $(\mathrm{r}=0.893)$, capítulos $(\mathrm{r}=0.851)$ y ponencias $(\mathrm{r}=0.941)$, lo cual implica que son estos productos los que impactan fuertemente en la producción total. Los artículos publicados con financiamiento y las direcciones de tesis no mostraron relación con otros productos. Los artículos se correlacionaron muy significativamente con los artículos en colaboración $(\mathrm{r}=0.799)$, lo cual indica que una buena proporción de los artículos se publican en colaboración con otros.

Hubo correlaciones significativas y muy significativas entre la producción científica y los 73 reactivos del instrumento, pero con niveles de asociación entre bajos y medios (hasta un valor de $\mathrm{r}=0.5$ ), por lo cual se dividió la muestra en cuartiles según distintos criterios y posteriormente se realizaron comparaciones de medias para cubrir parcialmente la comprobación de las hipótesis. Además, esto indica la necesidad de seguir investigando estos aspectos, con una muestra más numerosa y diversa de profesores, para determinar si se confirman los bajos niveles de asociación o si por el contrario estos incrementan.

Probamos correlacionar las variables del instrumento de Caprara et al. (2005) de actitudes prosociales, encontrando correlaciones significativas con alto nivel de relación (hasta un valor de $\mathrm{r}=0.7$ ), lo cual comprueba una gran consistencia interna y confiabilidad en cuanto al uso de dicho instrumento para estudiar las actitudes prosociales, incluso en un contexto educativo mexicano. En cuanto a las correlaciones entre los ítems que integraron la parte original del instrumento empleado (es decir, todos los reactivos, excepto los de Caprara et al., 2005), encontramos correlaciones entre todos estos, las cuales fueron de significativas a muy significativas y con niveles de asociación de altos a muy altos (entre 0.70 y 0.90 empleando el coeficiente de Pearson).

La comprobación de hipótesis de esta investigación se puede resumir en: I) el grupo de doctores que tiene la mayor producción global obtuvo calificaciones más bajas en las seis dimensiones prosociales; II) los investigadores más jóvenes son quienes han publicado la mayor cantidad de artículos y obtuvieron las mejores puntuaciones en las características prosociales; III) los investigadores que tienen la mayor cantidad global de producción científica tuvieron una calificación en motivación intrínseca ligeramente superior; IV) quienes obtuvieron su grado de doctor en el extranjero concentran más de la mitad de la producción y seis de ellos entraron en el SNI, y v) los miembros del SNI también concentran más de la mitad de la producción.

\section{DISCUSIÓN}

Los profesores encuestados se calificaron de manera similar en cinco de las seis dimensiones de conducta prosocial estudiadas, excepto en motivación extrínseca. Esta excepción podría indicar que los profesores sienten que los apoyos y reconocimientos de tipo moral, financiero y de recursos son limitados. Esto es evidenciable en los re- 
cortes presupuestarios de los últimos años que han sufrido las universidades públicas mexicanas, particularmente en los programas de estímulos a los profesores y a las iniciativas de apoyo a la investigación (Aristegui Noticias, 2019; Guglielmi, 2019). Sin embargo, su autoestima, autorregulación y motivación intrínseca parecen ser fuerzas importantes que impulsan la producción científica, lo cual es evidenciable en sus respuestas a las razones por las cuales investigan, ya que aquellas mejor calificadas fueron en su mayoría aquellas relacionadas con cuestiones intrínsecas y prosociales, como la satisfacción personal, el avance de su disciplina, compartir conocimiento y formar nuevos investigadores. En cambio, las razones calificadas más bajas correspondieron principalmente a cuestiones extrínsecas, como los apoyos y reconocimientos, obtención de fondos o requisitos laborales. Es importante señalar que, para que funcione la motivación intrínseca y el individuo pueda exhibir mejores conductas prosociales, este requiere de cierta autonomía para realizar sus labores (Schultz, Jones-Walker y Chikkatur, 2008). Sin embargo, es necesario contar con condiciones extrínsecas mínimas, como estar inmersos en entornos que favorezcan la colaboración, estímulos para realizar labores de investigación, contar con tiempo para investigar, así como buenas condiciones de seguridad y salud. Las debilidades en los apoyos se reflejan en los factores peor calificados, como el reconocimiento institucional, que además de los estímulos, también se relaciona con la promoción, difusión y reconocimiento de la labor de investigación de los profesores. Otros factores calificados de manera baja incluyeron la propia organización administrativa de la institución y la oferta de capacitación pertinente. Además, los profesores indicaron que contar con tiempo para investigar es frecuentemente importante, lo cual se relacionó con sus respuestas al respecto de que con poca frecuencia la investigación se ve favorecida positivamente por las cargas laborales, lo que indicaría que estas son altas y dificultarían dedicar tiempo a la investigación.

La fuerte motivación intrínseca y el valor que otorgan los profesores a su autonomía conforman un delicado equilibrio que les ha permitido realizar cierta producción científica. Sin embargo, tal equilibrio puede ser perjudicado por los factores extrínsecos calificados de una manera baja, ya que los problemas con la motivación extrínseca pueden terminar perjudicando la motivación intrínseca (Ryan y Deci, 2000).

Donde la muestra resultó más heterogénea y se exhibieron las brechas más amplias fue en los indicadores de producción científica. Los productos que los doctores afirmaron tener para el periodo estudiado (2016-2018) sumaron 1,197. Las ponencias fueron los productos más abundantes, con un total de $373(31.16 \%$ del total de productos), luego tenemos 205 capítulos de libro (17.13\%), 181 direcciones de tesis en proceso y concluidas durante el periodo (15.12\%), 155 artículos publicados en colaboración (12.95\%), 151 artículos arbitrados (12.61\%), 79 libros (6.60\%), 42 proyectos de investigación con financiamiento $(3.51 \%$ ) y 11 artículos publicados con financiamiento $(0.92 \%)$. 
Los profesores calificaron su desempeño de manera más baja respecto a sus pares, lo cual indicaría un nivel general de modestia. Según Bandura (1997), cada individuo desarrolla la convicción de que puede realizar exitosamente la conducta y hay percepciones, juicios y creencias que podrían ayudar a elevar las capacidades propias. Las creencias sobre las habilidades propias están asociadas directamente con el locus de control en relación a la creencia de poder o no realizar una determinada acción (Visdómine-Lozano y Luciano, 2006). Este tema también se relaciona con la autoestima (Caballo y Salazar, 2018; De Mézerville, 2004), la necesidad de autosuperación (Cardelle-Elawar y Sanz, 2010) y la competencia social, que beneficia la actitud prosocial del individuo (Caprara et al., 2005) y también le permite lograr una mejor integración en su ámbito de desempeño (Ryan y Deci, 2000).

Sin embargo, en los aspectos básicos relacionados con la realización de investigaciones los profesores se calificaron con una puntuación relativamente buena. Los profesores señalaron que la capacitación está entre los factores que de manera poco frecuente estimulan la investigación, es decir, lo estarían percibiendo como una necesidad, por lo cual la capacitación disponible pudiera no ser pertinente para desarrollar mayores habilidades de investigación. Adicionalmente, la voluntad para aprender lo que no saben sobre la investigación fue un aspecto bien calificado, por lo cual los profesores podrían ser receptivos a participar en un plan de capacitación sobre temas de investigación.

\section{CONCLUSIONES}

En la muestra se observó que las distribuciones resultaron bastante amplias en cuanto a edad, país de obtención de su doctorado, años de experiencia en investigación y años de experiencia en docencia. Lamentablemente, el grupo de hombres duplicó al grupo de mujeres, haciendo las comparaciones entre sexo más complicadas. Una buena cantidad de profesores tenían varias membresías, incluyendo PRODEP, pertenencia a un grupo disciplinar o cuerpo académico, e incluso un tercio de la muestra formaba parte del SNI, pero desafortunadamente cuatro miembros del SNI de la FFyL no participaron en la encuesta aplicada.

Las mujeres tuvieron puntuaciones ligeramente superiores a los hombres en los ítems de Caprara et al. (2005), por lo cual especulamos que las mujeres presentaron una mayor tendencia hacia las conductas prosociales. Dentro de los resultados obtenidos en el apartado sobre las razones por las cuales investigar se observó una mayor propensión asociada a la motivación extrínseca para llevar a cabo las acciones investigativas. Sobre los factores que estimulan positivamente la propia investigación y producción se observaron las medias más bajas del instrumento, lo cual hace pensar que los participantes de alguna manera sintieron incomodidad en esta sección, presuntamente debido a una falta de apoyo real o a características y procesos institucionales. Esto también se relacionó con la baja calificación de la dimensión de motivación extrínseca, señalada anteriormente. 
En la sección de autorregulación del instrumento, en su primera parte sobre la apreciación del propio desempeño y disposición destacó cierta apertura de los doctores hacia la formación permanente como investigadores. En cuanto a su desempeño en comparación con sus pares, se vieron reforzadas las tendencias de la parte anterior. Por la comparación de las puntuaciones entre las dos secciones, se observó que los encuestados percibieron que sus propias habilidades y disposición eran ligeramente inferiores a las de sus pares.

La mayor parte de la producción resultó ser de los hombres, sin embargo, esto se debe principalmente a las diferencias entre el número de mujeres y hombres encuestados, que fueron 10 mujeres y 21 hombres. Las mujeres resultaron tener el $34 \%$ de la producción, quizás en un grupo más equitativo en cuanto a sexo podría encontrarse que la producción entre sexos sea también más equilibrada.

Con respecto a la producción de los encuestados según el país donde obtuvieron su título de doctorado, se observó que quienes estudiaron en el extranjero poseían más de la mitad de la producción (54.39\%) frente a quienes lo hicieron en México (45.61\%). Esto fue un resultado relevante, ya que estos dos grupos eran casi iguales en número, los primeros fueron 16 y los segundos 15. En líneas generales, entre la producción científica y los 73 reactivos del instrumento, a pesar de que los niveles de asociación en las correlaciones resultaron bajos, las tendencias indicaron que es posible que mientras más publiquen los profesores, estos podrían ir disminuyendo sus puntuaciones en la dimensión de empatía, la cual está vinculada a conductas prosociales y a la motivación intrínseca.

La experiencia con el instrumento utilizado en esta investigación fue positiva, ya que se trató de un instrumento fuertemente fundamentado en las fuentes especializadas, además de haber arrojado altos valores cuando se calculó el alfa de Cronbach. A pesar de que se obtuvieron correlaciones más bajas y en menor cantidad de lo esperado, debido a la alta dispersión de algunos datos, consideramos que este instrumento es valioso para una aplicación a un grupo más amplio y diverso de doctores.

En una futura investigación en que se aplique este instrumento y se encuentren correlaciones a un mayor nivel de asociación, se debe aplicar regresión para predecir el nivel de las variables de características prosociales para incrementar la producción científica. Luego, se podría generar un modelo de ecuaciones de regresión, las cuales conducirían a pruebas confirmatorias que utilicen ecuaciones estructurales y puedan otorgar aún mayor validez y utilidad al modelo teórico-metodológico utilizado, para así explicar con mayor exactitud el rol y asociación con altos índices de sensibilidad de ajuste de las variables psicológicas que afecten la producción científica.

Esta línea de investigación puede aportar estrategias psicológicas necesarias para fomentar la motivación docente, las cuales pudieran no ser implementadas de manera explícita o intencional, especialmente en la formación y en el impulso de investigadores. Existe un área de oportunidad amplia para implementar el punto de vista psicológico en el fomento de la productividad científica. 


\section{REFERENCIAS}

Aristegui Noticias (2019). Recortes a CONACyT pueden colapsar centros de investigación en México: investigadores. Recuperado de: https://aristeguinoticias.com/2205/mexico/recortes-aconacyt-pueden-colapsar-centros-de-investigacion-en-mexico-investigadores.

Bandura, A. (1997). Self-efficacy: The exercise of control. Nueva York, EEUU: W. H. Freeman/ Times Books/Henry Holt \& Co.

Caballo, V., y Salazar, I. (2018). La autoestima y su relación con la ansiedad social y las habilidades sociales. Psicología Conductual, 26(1), 23-53. Recuperado de: https:// dialnet.unirioja. es/servlet/articulo?codigo $=6378325$.

Caprara, G., Steca, P., Zelli, A., y Capanna, C. (2005). A new scale for measuring adults' prosocialness. European Journal of Psychological Assessment, 21(2), 77-89. DOI: https://doi. org/10.1027/1015-5759.21.2.77.

Cardelle-Elawar, M., y Sanz, M. (2010). Looking at teacher identity through self-regulation. Psicothema, 22(2), 293-298. Recuperado de: http://www.psicothema.com/PDF/3729.pdf.

De Mézerville, G. (2004). Ejes de salud mental: los procesos de autoestima, dar y recibir afecto y adaptación al estrés. Trillas.

Guglielmi, G. (2019). Mexican science suffers under debilitating budget cuts. Nature, 572, 294-295. DOI: https://doi.org/10.1038/d41586-019-02332-x.

Inzunza, A. (2017). Conversando con Luis Arturo Godínez: el SNI ha propiciado cambios institucionales y culturales. Revista Forum: Noticias del Foro Consultivo Cientifico y Tecnológico, (31), 8-17. Recuperado de: https://www.foroconsultivo.org.mx/forum/2017_diciembre/ mobile/index.html.

Ladd, J., Lappé, M., McCormick, J., Boyce, A., y Cho, M. (2009). The 'how' and 'whys' of research: Life scientists' views of accountability. Journal of Medical Ethics, 35(12), $762-767$. DOI: https://doi.org/10.1136/jme.2009.031781.

Landini, F. (2015). Contributions of community psychology to rural advisory services: An analysis of Latin American rural extensionists' point of view. American Journal of Community Psychology, 55(3), 359-368. DOI: https://doi.org/10.1007/s10464-015-9712-4.

Landini, F., Olivera, A., y De Hegedüs, P. (2017). Psychology's contributions to extension: State of the art and calls to action. Journal of Extension, 55(4), 1-6. Recuperado de: https:// joe.org/joe/2017august/pdf/JOE_v55_4comm2.pdf.

Martínez, N. (2018). Más producción científica, urgen a universidades. En Consorcio Nacional de Recursos de Información Científica y Tecnológico (CONRICyT). Noticias y avisos. Recuperado de: http://conricyt.mx/noticias-y-avisos/mas-produccion-cientifica-urgen-a-universidades. htm.

Mattedi, M., y Spiess, M. (2017). A avaliação da produtividade científica. História, Ciências, SaúdeManguinhos, 24(3), 623-643. DOI: https:// doi.org/10.1590/s0104-59702017000300005.

Mulligan, A., y Mabe, M. (2011). The effect of the internet on researcher motivations, behaviour and attitudes. Journal of Documentation, 67(2), 290-311. DOI: https://doi. org/10.1108/00220411111109485.

Öcshner, A. (2013). Introduction to scientific publishing: Backgrounds, concepts, strategies. Heidelberg, Alemania: Springer.

Offutt, J. (2011). Editorial: What is the purpose of publishing? Software: Testing, Verification and Reliability, 21(4), 265-266. DOI: https://doi.org/10.1002/stvr.468. 
Patterson-Hazley, M., y Kiewra, K. (2013). Conversations with four highly productive educational psychologists: Patricia Alexander, Richard Mayer, Dale Schunk, and Barry Zimmerman. Educational Psychology Review, 25(1), 19-45. DOI: https://doi.org/10.1007/ s10648-012-9214-y.

Reyes, M., y Perales, M. (2016). Research self-efficacy sources and research motivation in a foreign language university faculty in Mexico: Implications for educational policy. Higher Education Research and Development, 35(4), 800-814. DOI: https://doi.org/10.1080/0729 4360.2015.1137884.

Rodríguez, C. (2016). El Sistema Nacional de Investigadores en números. México: Foro Consultivo Científico y Tecnológico. Recuperado de: http:/ /www.foroconsultivo.org.mx/libros_editados/SNI_en_numeros.pdf.

Ryan, R., y Deci, E. (2000). Intrinsic and extrinsic motivations: Classic definitions and new directions. Contemporary Educational Psychology, 25(1), 54-67. DOI: https://doi.org/10.1006/ ceps.1999.1020.

Sánchez, V. (2017). Crece 23 por ciento la producción científica en México. En CONACyT Agencia Informativa. Politica cientifica. Recuperado de: http://www.conacytprensa.mx/index.php/ sociedad/politica-cientifica/18011-crece-23-por-ciento-produccion-cientifica-mexico.

Schultz, K., Jones-Walker, C., y Chikkatur, A. (2008). Listening to students, negotiating beliefs: Preparing teachers for urban classrooms. Curriculum Inquiry, 38(2), 155-187. DOI: https://doi.org/10.1111/j.1467-873X.2007.00404.x.

Siciliano, M., Welch, E., y Feeney, M. (2018). Network exploration and exploitation: Professional network churn and scientific production. Social Networks, 52, 167-179. DOI: https://doi.org/10.1016/j.socnet.2017.07.003.

Swan, A., y Brown, S. (2005). Open access self-archiving: An author study. Truro, Reino Unido: Key Perspectives. Recuperado de: http:// cogprints.org/4385/1/jisc2.pdf.

Visdómine-Lozano, C., y Luciano, C. (2006). Locus de control y autorregulación conductual: revisiones conceptual y experimental. International Journal of Clinical and Health Psychology, 6(3), 729-751. Recuperado de: https:/ / dialnet.unirioja.es/servlet/articulo?codigo $=2110683$.

Wigfield, A. (2002). Expectancy-value theory of achievement motivation. Journal of Asynchronous Learning Network, 6(1), 68-81. DOI: https://doi.org/10.1006/ceps.1999.1015.

Cómo citar este artículo:

Agredo-Machin, D., Chávez-Valdez, S. M., y Romo-González, J. R. (2020). Características prosociales y producción científica de académicos de la Facultad de Filosofía y Letras de la Universidad Autónoma de Chihuahua. RECIE. Revista Electrónica Científica de Investigación Educativa, 5(1), pp. 255-266. doi: doi.org/10.33010/ recie.v5i1.992 